\title{
Temática del proyecto de ley y cohesión en la votación de las coaliciones en el Congreso de Chile, 2010-2017
}

\author{
Bill subject and voting cohesion by coalitions \\ in the Chilean Congress, 2010-2017
}

\author{
VERÓNICA FIGUEROA-GUTIÉRREZ \\ Universidad Diego Portales
}

PATRICIO NAVIA

Universidad Diego Portales

New York University

\section{Cómo citar/Citation}

Figueroa Gutiérrez, V. y Navia, P. (2021). Temática del proyecto de ley y cohesión en la votación de las coaliciones en el Congreso de Chile, 2010-2017. Revista Española de Ciencia Política, 57, 191-217. Doi: https://doi.org/10.21308/recp.57.07

\section{Resumen}

Estudios sobre la cohesión de legisladores en las votaciones en sala han reportado un efecto de pertenecer al oficialismo, del origen e importancia del proyecto, del ciclo electoral y otros aspectos del diseño institucional. Pero la teoría también asocia la cohesión en las votaciones a la temática del proyecto, un aspecto que ha sido insuficientemente abordado en estudios sobre cohesión partidista en democracias presidencialistas de América Latina. Este artículo somete a prueba cuatro hipótesis que incluyen el efecto de la temática en la cohesión de las votaciones de las coaliciones en las 6468 votaciones en sala en la Cámara de Diputados de Chile entre los ańos legislativos 2010 y 2017. Los legisladores de la coalición de derecha se cohesionan más en temas de defensa y seguridad ciudadana. En contra de las expectativas, los de izquierda no se cohesionan más en temas de protección social o derechos humanos. Los legisladores del oficialismo se cohesionan menos en votaciones sobre presupuesto. La temática del proyecto parece importar más a la derecha que a la izquierda, aunque especulamos que esto puede deberse en Chile a que la coalición de derecha está compuesta por un número menor de partidos con mayor homogeneidad ideológica.

Palabras clave: comportamiento legislativo, votaciones nominales, cohesión, temática de la ley, ideología, Chile.

\section{Abstract}

Studies on the cohesion by legislators in roll call votes have reported an effect of being a member of the ruling coalition, the origin and relevance of the bill, the electoral cycle and other 
institutional design features. But the theory also links voting cohesion to the subject of the bill, an issue not sufficiently addressed on studies on party-level cohesion in Latin American presidential democracies. This article tests four hypotheses including the subject of the bill on the 6468 roll call votes in the Chamber of Deputies of Chile between 2010 and 2017 legislative years. Rightwing legislators are more cohesive in roll call votes on issues related to defense and citizen security. Contrary to expectations, leftwing legislators are not more cohesive on social issues or human rights. Ruling coalition legislators are less cohesive on budget votes. The subject of the bill seems more important for rightwing legislators than leftwing ones; although it is speculated that this might be due in Chile to the fact that the rightwing coalition is comprised by a smaller number of parties which shares more ideological homogeneity.

Keywords: legislative behavior; roll call voting; cohesion; bill subject; ideology; Chile.

\section{INTRODUCCIÓN}

La condición de oficialismo, el origen y la importancia del proyecto que se vota, el ciclo electoral y otros aspectos del diseño institucional influyen en la cohesión en votaciones nominales de los legisladores de distintos partidos en democracias presidencialistas. Ahora bien, como los partidos tienen distintos niveles de compromiso con posiciones ideológicas y sus legisladores defienden esas posiciones con distinta intensidad, la temática del proyecto que se vota pudiera afectar también a la cohesión de un partido o coalición en sus votaciones nominales registradas en la legislatura. Es más, en democracias presidencialistas con coaliciones multipartidistas estables, la presencia de temas ideológicamente contenciosos para una coalición multipartidista puede generar tensiones que se vean reflejadas en la cohesión en la votación de los legisladores que militan en los distintos partidos que forman cada coalición. De ahí que este artículo aborde el efecto de la temática de los proyectos de ley que se votan en sala sobre la cohesión que muestran los legisladores que militan en los distintos partidos que componen dichas coaliciones.

Usamos el caso de Chile, una democracia presidencialista con un sistema de partidos institucionalizado que, entre 1990 y 2017, se organizó en torno a dos coaliciones multipartidistas estables. Con regularidad, la temática de los proyectos de ley que se discutían en el Congreso produjo tensiones en el interior de cada coalición, producto de posiciones divergentes de sus partidos miembros. Por ejemplo, en julio de 2017, después de haber sido aprobado en el Senado, un proyecto de ley para despenalizar el aborto bajo tres causas fue votado en la Cámara de Diputados, donde se produjo una división entre legisladores de distintos partidos de la coalición oficialista mayoritaria, la centroizquierdista Nueva Mayoría (NM). Si bien los partidos de la coalición habían anunciado su apoyo a esta iniciativa que, además, era apoyada por la presidenta Michelle Bachelet, líder de la NM, tres diputados del Partido Demócrata Cristiano (PDC) se negaron a apoyar el proyecto, dos de ellos votando en contra y uno absteniéndose. El proyecto no fue aprobado por un voto. El diputado del PDC Marcelo Chávez, quien se abstuvo, fue 
sindicado como responsable del rechazo del proyecto en la Cámara y, por lo tanto, de que el proyecto tuviera que ir a una comisión de conciliación entre ambas cámaras. Eventualmente, el proyecto fue promulgado, pero las discrepancias sobre este proyecto entre diputados de la coalición gobernante hicieron que el oficialismo no pudiera votar de forma cohesionada ese proyecto.

Aquí evaluamos el efecto que tiene la temática del proyecto de ley —identificada a partir de la comisión permanente que tramitó el proyecto- sobre la cohesión en la votación en sala de los contingentes de cada coalición multipartidista. Para ello, usamos las 6468 votaciones efectuadas en la Cámara de Diputados en Chile en los dos periodos legislativos comprendidos entre los años legislativos 2010 y 2017. A partir de una discusión teórica sobre los principales determinantes de la cohesión de los legisladores en sus votaciones en sala, postulamos cuatro hipótesis que asocian la cohesión en la votación de proyectos con: a) el momento en el ciclo electoral en que se realiza la votación; b) la condición de oficialismo; c) la interacción entre la ideología de cada coalición, y d) la temática de los proyectos que se votan. Después presentamos el caso, discutiendo el proceso legislativo en Chile y los principales aspectos contextuales del periodo. A continuación, procedemos a presentar la metodología y los datos y a realizar un análisis inferencial. En las conclusiones discutimos también las implicaciones que este estudio tiene para entender mejor la varianza en la cohesión de las coaliciones multipartidistas en las votaciones en democracias presidencialistas.

\section{DETERMINANTES DE LA COHESIÓN EN VOTACIONES EN SALA}

En general, el comportamiento de los legisladores responde a su deseo de buscar la reelección o mejorar en sus carreras aspirando a una posición que consideren más importante (Schlesinger, 1966). De ahí que los estudios sobre su comportamiento se centren en variables a nivel individual (Özbudun, 1970; Poole y Rosenthal, 2001). Pero como los legisladores necesitan del apoyo de sus partidos, votantes o financiadores para ser reelegidos o progresar en sus carreras, deben tomar en cuenta sus intereses en su comportamiento legislativo (Carey, 2007; Janda, 1980). A su vez, las reglas de funcionamiento del Congreso también influyen sobre el comportamiento de los legisladores (Cox y McCubbins, 2007). Por eso, la combinación de incentivos individuales y legados partidistas ayuda a explicar la cohesión en la votación en sala de los partidos (Kitschelt y Smyth, 2002) y, por extensión, en las coaliciones en los países con sistemas multipartidistas. Por ello, aquí abordamos tanto incentivos sistémicos derivados de las reglas electorales como incentivos individuales asociados a las posiciones ideológicas de los legisladores y de sus partidos para dar cuenta de los determinantes de la cohesión en las votaciones en sala de las coaliciones multipartidistas.

Las reglas electorales influyen en el comportamiento de los legisladores y en la cohesión de los partidos en sus votaciones en sala. Por ejemplo, en sistemas uninominales o de representación proporcional con lista abierta, los legisladores tienen incentivos para cultivar un voto personal (Carey y Shugart, 1995). Eso los puede llevar a 
mostrar menor cohesión con sus partidos cuando las preferencias de sus electores y de sus partidos difieren (Carey, 2007). Por su parte, en sistemas donde los líderes partidistas controlan la nominación de candidatos, los legisladores podrían mostrar mayor disposición a votar de la misma forma que el liderazgo de sus partidos para optimizar su probabilidad de volver a ser nominados (íd.). Estudios previos identifican un efecto combinado de las reglas electorales con los procedimientos y normas que implementan los partidos para disciplinar a sus miembros y, a la vez, permitirles que usen las herramientas disponibles para avanzar sus propias carreras e intereses (íd.; Shepsle y Weingast 1994; Cox y McCubbins, 2005). En sistemas multipartidistas con partidos pragmáticos es más fácil construir coaliciones de gobierno que, a su vez, influyen en la cohesión de sus legisladores (Morgenstern, 2004).

Las reglas que rigen la relación Ejecutivo-Legislativo y el funcionamiento del Congreso también impactan en la cohesión de las votaciones en sala de un partido o coalición multipartidista (Tsebelis, 2002; Morgenstern, 2004; Figueiredo y Limongi, 2000). En América Latina, los presidentes en general poseen poderes proactivos - como la capacidad de presentar proyectos de ley, en algunos casos con iniciativa exclusiva, e introducir enmiendas en cualquier momento de la tramitación de un proyecto-, mientras que las legislaturas poseen poderes reactivos que les permiten negociar con el Ejecutivo, para así incorporar sus prioridades en los proyectos que se tramitan (Cox y Morgenstern, 2001; Mainwaring y Shugart, 2002). Los Gobiernos tienden a usar esos poderes proactivos para inducir mayor disposición de los legisladores oficialistas a votar a favor de sus proyectos prioritarios (Figueiredo y Limongi, 2000). Los partidos usan incentivos positivos y negativos para promover la cohesión en cómo votan sus legisladores (Sieberer, 2006). El poder que tienen los partidos permite a sus líderes premiar a legisladores leales con membresía en comisiones más importantes y con acceso a recursos para sus distritos o bases de apoyo (Carey, 2009).

La interacción entre la influencia del Ejecutivo en el comportamiento de los legisladores y las reglas del funcionamiento del Congreso se evidencia a través del ciclo electoral. En el primer año de gobierno, por el efecto de luna de miel, hay un mayor apoyo a la agenda legislativa del presidente, lo que redunda en una cohesión mayor de sus aliados en el Congreso (Canes-Wrone y de Marchi, 2002: Alemán y Calvo, 2008; Alemán y Navia, 2009). Como los presidentes también tienden a ser más populares en su primer año de gobierno, deberíamos esperar que los legisladores oficialistas - ya sea del partido del presidente o de los partidos que forman la coalición multipartidista de gobierno - muestren mayor cohesión, especialmente en proyectos que son de interés del presidente. Adicionalmente, precisamente porque al finalizar sus periodos los presidentes salientes tienen menos influencia sobre los legisladores oficialistas — que están más preocupados de su propia reelección o de sus carreras futuras - hay un efecto de último año que reduce el apoyo a las iniciativas del presidente, especialmente en el oficialismo (Campos-Parra y Navia, 2017; Martínez y Navia, 2019). Por tanto, como se espera que el oficialismo sea más cohesionado en el primer año que en el último año en sistemas presidenciales, nuestra primera hipótesis sostiene que: 
H1. En Chile, hay mayor cohesión en la votación en sala de los legisladores de la coalición oficialista en votaciones al comienzo del periodo que en el último año.

Reflejando la importancia del diseño institucional, las investigaciones sobre cohesión en las votaciones en democracias presidenciales de América Latina sostienen que los partidos, o en los casos correspondientes las coaliciones oficialistas, registran mayor cohesión que los de oposición, aunque las reglas electorales y la cultura partidista impactan también en la cohesión (Morgenstern, 2004; Alemán y Calvo, 2008; Alemán, 2013). La cohesión en sistemas multipartidistas, como el de Brasil, se ha asociado a la capacidad de los presidentes de generar más apoyo a sus iniciativas al incorporar a distintos partidos al Gabinete (Amorim Neto, 2000). En aquellos sistemas en los que el Ejecutivo tiene importantes poderes y atribuciones, puede ejercer más influencia sobre los legisladores oficialistas y así lograr que estos se cohesionen más que los de oposición. Si esto es cierto, esa mayor cohesión debería verse claramente reflejada en los asuntos que el Ejecutivo considera prioritarios. Por eso, teniendo en cuenta que las cuestiones de presupuesto, y en general todas aquellas que implican gasto público, son prioritarias para cualquier Gobierno, nuestra segunda hipótesis sostiene que:

H2. Los legisladores de la coalición oficialista muestran más cohesión en votaciones sobre presupuesto y en proyectos de ley referidos a la Comisión de Hacienda que los legisladores de otras coaliciones.

Pero las diferencias entre coaliciones no responden solo a la condición de oficialismo u oposición. La ideología también ayuda a entender por qué algunos partidos o coaliciones priorizan determinados asuntos. La ideología es un conjunto de proposiciones coherentes internamente que dan guía al comportamiento humano (Hinich y Munger, 1996), generando una unión entre lo que piensa el individuo y su accionar (Kerlinger, 1984). Normalmente, la ideología se mide en una escala de izquierda a derecha, pero también se pueden usar otras escalas, como liberal-conservador.

En sistemas de partidos institucionalizados, los partidos a menudo se identifican con una ideología determinada, con la que comulgan sus integrantes (Van der Brug, 2004). Por eso, debiéramos esperar que los valores y actitudes de los legisladores y de sus partidos también impacten en el comportamiento legislativo (Londregan, 2000). Es más, la ideología del partido influye sobre la capacidad de los líderes partidistas de cohesionar a sus contingentes legislativos (Close, 2018).

La importancia que dan los partidos a ciertos temas genera un efecto de transversalidad (Green, 2007). De hecho, Stokes (1963; 1992) postuló tempranamente los términos valence issue y position issue —el término valence, usado inicialmente por Downs, se traduce normalmente como «transversalidad» (Astudillo y Rodón, 2013: 10) —. Por eso, en un valence issue — una cuestión que genera transversalidad- existe consenso sobre los fines. En cambio, en un position issue existen diversos puntos de vista sobre un determinado tema. Cuando se tramitan proyectos de ley, la discusión se 
centra en esas dos dimensiones, en tanto el Congreso legisla sobre algún asunto cuando existe un consenso transversal en la necesidad de reformar ciertas políticas públicas (valence) pero hay disenso en cómo debería cambiar el statu quo (position). Así, por ejemplo, cuando hay crisis económica, la creación de empleo pudiera adquirir más transversalidad que la discusión sobre los derechos reproductivos de la mujer.

Existen ciertos temas que se emparentan, por tradición o pragmatismo, con ideologías. Por ejemplo, el concepto de issue ownership —o propiedad de un tema- sostiene que hay asuntos que se asocian a partidos, líderes o corrientes ideológicas en las campañas (Petrocick, 1996) y en el proceso político (Bélanger, 2000; Stubager y Slothuus, 2013). La transversalidad interactúa con el issue ownership - o dominancia sobre un tema-. Por ejemplo, puede haber un acuerdo transversal en los partidos para reformar el sistema de pensiones, pero hay discrepancias sobre en qué dirección reformarlo. Por eso, cuando posee propiedad sobre un tema, un partido o candidato tiene una ventaja comparativa cuando se discuten proyectos relacionados con ese tema. Así, el issue ownership debiera también tener un efecto sobre la cohesión de los miembros de un partido cuando votan sobre temas respecto a los que el partido ejerce dominancia.

Ahora bien, por la importancia que la gente atribuye al buen desempeño de la economía, todos los partidos aspiran a ser dominantes en el manejo económico (Egan, 2013; Belánger y Nadeau, 2015). De ahí que, aunque lo intente, ningún partido puede ejercer, al menos de forma permanente, dominancia sobre el manejo de la economía. En otras palabras, la economía es un tema que genera transversalidad. En el caso de los sistemas presidenciales, podemos suponer que el partido o coalición que controla el Gobierno logró convencer al electorado de su mayor capacidad para ejercer un buen manejo económico en el periodo actual —y por eso, está en el poder-. En consecuencia, parece razonable esperar que los legisladores oficialistas muestren más cohesión en votaciones sobre cuestiones de política económica y de presupuesto. Pero en otras dimensiones, debiéramos esperar diferencias entre legisladores de izquierda y derecha en sus prioridades — y en la dominancia — sobre distintos temas.

Los estudios sobre issue ownership asocian a los partidos de derecha con una mejor capacidad para combatir la delincuencia o generar empleo (Seeberg, 2017; Belánguer y Nadeau, 2015). A su vez, la derecha en general demuestra más aceptación de lo natural, la costumbre y la tradición, mientras que la izquierda pone el foco en la igualdad (Bobbio y Squella, 1995). McClosky y Zaller (1984) definen a los legisladores y a las políticas como liberales cuando buscan promover valores como la igualdad, la ayuda a los más desvalidos, la tolerancia y la promoción de reformas sociales, y como conservadoras cuando priorizan el orden público, los negocios y la defensa nacional. Por su parte, históricamente, a los partidos de izquierda se les atribuye la defensa de políticas que privilegian los intereses de las clases baja y media, como el aumento de gasto social o medidas redistributivas (Hicks y Swank,1992; Korpi y Palme, 2003; Allan y Scruggs, 2004). Otros han mostrado que los partidos de izquierda ejercen dominancia sobre asuntos de políticas sociales (Seeberg, 2017; Belánguer y Nadeau, 2015). Los votantes de izquierda tienden a estar en los sectores más vulnerable o de menores ingresos, por lo que se infiere que en periodos de crisis los partidos 
de izquierda promoverán políticas que aumenten el gasto social (Ahrend et al., 2011). Por lo tanto, nuestra tercera hipótesis postula que:

H3. Independientemente de si ocupan la posición de oficialismo u oposición, los legisladores de la coalición de derecha en Chile se cohesionan más en votaciones sobre temas de defensa y seguridad ciudadana, mientras que los de la coalición de izquierda lo hacen sobre temas de protección social.

Las diferencias ideológicas entre izquierda y derecha también se manifiestan de forma contextual a los debates en cada país. Desde 1990, la dinámica política de Chile se caracterizó por mayores acuerdos entre las coaliciones de centroizquierda y de derecha en temas económicos que en materias de diseño institucional (Sehnbruch y Siavelis, 2014). La estructura económica heredada del régimen militar se fue consolidando durante los primeros Gobiernos de la Concertación (Ffrench-Davis, 2002), y destacó la focalización del gasto en los sectores más vulnerables, la apertura económica y el incremento en la productividad, lo que hizo posible mayor desarrollo económico, mejoras en los salarios reales y disminución sustantiva de la pobreza (Larraín y Vergara, 2000; Meller, 2005).

Pero mientras hubo grandes acuerdos que permitieron estabilidad en las políticas económicas, hubo discrepancia sobre el mantenimiento de enclaves autoritarios. De hecho, la transición a la democracia obligó a los partidos de centroizquierda a aceptar la Constitución de 1980, promulgada en dictadura — aunque desde su llegada al poder en 1990, la coalición centroizquierdista Concertación intentó eliminar los enclaves autoritarios de la Constitución (Garretón, 2003) — , pero los altos quórums que existen en la Constitución — considerados, por muchos, en sí mismos como enclaves autoritarios - hacen que, para cambiar el texto, se requiera de mayorías calificadas en el Congreso (Fuentes, 2015). Por eso mismo, dado su interés histórico en eliminar los enclaves autoritarios y la necesidad de alcanzar mayorías amplias en el Congreso para lograrlo, esperamos que los legisladores de la coalición de centroizquierda se muestren especialmente cohesionados en votaciones sobre reformas constitucionales. A su vez, como los partidos de derecha que forman la coalición Alianza han discrepado respecto a qué modificaciones constitucionales corresponde realizar, esperamos que los legisladores de esa coalición muestren menor cohesión en ese tipo de votaciones.

Desde 1990, la Concertación también promovió una agenda en materia de derechos humanos para corregir y remediar las violaciones sistemáticas a los mismos cometidas en dictadura (Zalaquett, 2000), por lo que esa coalición logró ejercer dominancia en ese tema y, presumiblemente, mostró más cohesión en votaciones relacionadas con esos asuntos. A partir de esta discusión, planteamos la cuarta hipótesis, particular del caso de Chile, que sostiene que:

H4. En Chile, los legisladores de la coalición de izquierda se cohesionan más que los de la coalición de derecha en votaciones relacionadas con debates constitucionales y con temas de derechos humanos. 


\section{EL PROCESO LEGISLATIVO Y EL SISTEMA DE PARTIDOS EN CHILE}

El proceso legislativo en el Congreso bicameral de Chile es secuencial. Cuando ingresa un proyecto de ley en una Cámara y esta aprueba la idea de legislar, el proyecto se deriva a una comisión permanente que discute el proyecto y luego lo envía a la sala. Se pueden presentar indicaciones o enmiendas en cualquier momento de la tramitación. Si el proyecto es aprobado por la primera Cámara, pasa a la segunda para un proceso similar. Si esta modifica el proyecto, es enviado nuevamente a la primera. Si no hay acuerdo entre ambas cámaras, el proyecto pasa a una comisión mixta, compuesta por representantes de ambas cámaras que busca un acuerdo.

Hay tres tipos de mayorías calificadas para que un proyecto sea aprobado. El quorum simple requiere la mitad más uno de los diputados presentes para aprobar un proyecto. El quorum calificado requiere la mitad más uno de los diputados en ejercicio. Las leyes orgánicas constitucionales (LOC) solo pueden ser aprobadas por mayoría de 4/7 de los legisladores en ejercicio y las reformas constitucionales requieren de mayoría de $3 / 5$ o $2 / 3$.

Desde 1990, el sistema político chileno se ha ordenado en torno a dos coaliciones multipartidistas estables. La centroizquierdista Concertación y la centroderechista Alianza. La Concertación estaba formada por el Partido Socialista (PS), Partido Demócrata Cristiano (PDC), Partido por la Democracia (PPD) y el Partido Radical Social Demócrata (PRSD). Para las elecciones de 2013, la Concertación sumó al Partido Comunista (PC) y otros grupos menores, adoptando el nombre de NM —aquí usamos el término Concertación/NM para referirnos a esa coalición-. Por otro lado, la Alianza estaba compuesta por los partidos Unión Demócrata Independiente (UDI) y Renovación Nacional (RN). En 2013, esa coalición sumó otros partidos menores, el Partido Regionalista Independiente (PRI) y Evolución Política (EVOPOLI).

Después de veinte años en el poder de la Concertación, el agotamiento de sus elencos y su falta de renovación ofrecieron a la Alianza una oportunidad para llegar al poder en 2009, con un discurso de renovación de liderazgos personificado en su candidato Sebastián Piñera, cuya campaña puso el foco en el crecimiento económico, la creación de empleo y el combate a la delincuencia. No obstante, la lianza no alcanzó mayoría en ninguna de las cámaras, aunque en acuerdos con partidos minoritarios logró controlar la Cámara de Diputados. Para las elecciones de 2013, la candidata presidencial de la NM, Michelle Bachelet, ganó cómodamente en segunda vuelta. Su coalición, NM, obtuvo mayoría en ambas cámaras. Así, en los dos periodos presidenciales y legislativos que aquí estudiamos, hubo alternancia en el poder entre ambas coaliciones. Por su parte, a través de acuerdos con partidos menores, la Alianza logró controlar una mayoría en la Cámara de Diputados en 2010-2014, mientras que en 2014-2018 la Concertación —bajo el nuevo nombre Nueva Mayoría- logró un control mayoritario en la Cámara. La tabla 1 muestra la composición partidista y por coalición en la Cámara de Diputados para los dos cuatrienios legislativos que se iniciaban en marzo de cada año y concluían en marzo del año siguiente. 
Tabla 1.

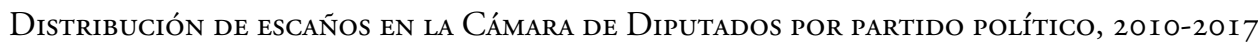

\begin{tabular}{|c|c|c|c|c|}
\hline \multirow{2}{*}{ Partido político } & \multicolumn{2}{|c|}{ Ańos legislativos 2010-2013 } & \multicolumn{2}{|c|}{ Ańos legislativos 2014-2017 } \\
\hline & \# diputados & $\%$ diputados & \# diputados & $\%$ diputados \\
\hline \multicolumn{5}{|c|}{ Concertación/NM } \\
\hline PS & 12 & 10 & 15 & 12.5 \\
\hline PDC & 19 & 15.8 & 21 & 17.5 \\
\hline PPD & 18 & 15.0 & 15 & 12.5 \\
\hline PC & 3 & 2.5 & 6 & 5.0 \\
\hline Otros & - & - & 4 & 3.3 \\
\hline PRSD & 5 & 4.2 & 6 & 5.0 \\
\hline Concertación/NM total & 57 & 47.5 & 67 & 55.8 \\
\hline \multicolumn{5}{|c|}{ Alianza } \\
\hline UDI & 38 & 31.6 & 29 & 24.2 \\
\hline $\mathrm{RN}$ & 17 & 14.2 & 19 & 15.8 \\
\hline Otros & 3 & 2.5 & 1 & 0.8 \\
\hline Alianza total & 58 & 48.3 & 49 & 40,8 \\
\hline Independientes y otros & 5 & 4.2 & 4 & 3.4 \\
\hline Total & 120 & 100 & 120 & 100 \\
\hline
\end{tabular}

Fuente: elaboración propia a partir de los datos extraídos de: www.servel.cl

\section{DATOS Y METODOLOGÍA}

Aunque a veces los conceptos de cohesión y disciplina legislativa se usan de forma indistinta (Toro, 2007), es preciso distinguirlos. La cohesión es la medida en que los miembros de un partido poseen preferencias semejantes que se ven reflejadas en su comportamiento (Carey 2002, 2007) —en este caso, en sus votaciones en sala_- En cambio, la disciplina se define en términos de obediencia de los legisladores al liderazgo del partido, que usa "garrotes» y «zanahorias» para castigar o premiar a militantes (íd.; Morgenstern, 2004). De hecho, cuando la cohesión de un partido es baja, la disciplina aparece como solución para inducir a la unidad partidaria (Hazan, 2003). Por su parte, hay estudios que analizan la unidad partidaria como coherencia, basándose en las posiciones declaradas de los legisladores en encuestas más que en sus votaciones en sala (Ruiz Rodríguez, 2006; Freidenberg et al., 2006). En lo que sigue, para unificar la terminología usamos el concepto de cohesión en votaciones en sala - o solo cohesión - para referirnos a cómo de unidos votan en sala los legisladores de cada coalición política.

Los estudios sobre cohesión a menudo utilizan las votaciones nominales en el Congreso de Estados Unidos (Poole y Rosenthal, 2001) y las votaciones partidarias 
para las legislaturas europeas (Hix, 2001). Usando los votos en sala (roll call), la forma más común de medir la cohesión es a través de los indicadores Unity y Rice (Rice, 1925; Carey, 2009). En ambos casos, se genera un parámetro que va de 0 a 1 -nula a completa cohesión-. Unity considera a los legisladores ausentes como votos en contra de la mayoría, mientras que Rice no considera a los legisladores ausentes, por lo que tiende a sobreestimar la cohesión. Para entender la diferencia entre ambos indicadores, supongamos una coalición con 49 diputados, en la que solo 41 se presentan a votar y todos votaron contra un proyecto. Mientras Rice reporta una cohesión perfecta, Unity reporta una cohesión de 0.84 (41 de 49 legisladores).

La variable dependiente en este estudio es la cohesión de los legisladores en sus votaciones en sala a nivel de coalición, no de partido político. Esto por dos razones. Primero, en sistemas multipartidistas la presencia de partidos con un número reducido de legisladores presenta problemas de sesgo al inflar artificialmente los resultados de cohesión para esos partidos (Desposato, 2005). De ahí que, para evitar ese sesgo, agrupamos a los partidos en coaliciones, que al ser menos numerosas y presentar estabilidad en el tiempo, reducen el riesgo de ese sesgo. Segundo, en Chile los partidos han formado coaliciones multipartidistas estables que a menudo son tratadas como partidos (Carey, 2002; Morgenstern, 2004; Alemán y Saiegh, 2007) —a la usanza de la también coalición multipartidista Frente Amplio en Uruguay-. Por eso, aquí calculamos los indicadores Rice y Unity para la Concertación/NM, Alianza, oficialismo y oposición.

La unidad de análisis es la votación en sala sobre un proyecto de ley. Analizamos las 6468 votaciones en sala en la Cámara de Diputados en los dos periodos legislativos comprendidos entre marzo de 2010 y marzo de 2018 — esto es, entre los ańos legislativos 2010 y 2017 — En ambos periodos, el Gobierno que asumió representaba alternancia en el poder. En 2010-2013, por primera vez desde el retorno de la democracia, gobernó la coalición de derecha. En 2014-2017, por primera vez desde el retorno de la democracia, la coalición de izquierda volvió al poder después de haber sido oposición. En Chile, los legisladores pueden votar a favor, en contra, abstenerse o parearse. Esta última opción implica votar presente, pero no emitir un voto, en tanto el legislador tiene un acuerdo con otro legislador de un partido político opuesto que los obliga mutuamente a no votar cuando el otro legislador está ausente. Aquí consideramos los pareos como ausencias. La tabla 2 muestra las votaciones por año legislativo durante el periodo.

TABLA 2.

Número de votaciones según año legislativo, Cámara de Diputados, 20io-20I7

\begin{tabular}{lccccc}
\hline Ańo legislativo & \# votaciones & \% & Periodo legislativo & \# votaciones & \% \\
\hline $\mathbf{2 0 1 0}$ & 664 & 10.3 & & & \\
\hline $\mathbf{2 0 1 1}$ & 746 & 11.5 & & & \\
\hline $\mathbf{2 0 1 2}$ & 828 & 12.8 & $2010-2013$ & 3189 & 49.3 \\
\hline $\mathbf{2 0 1 3}$ & 951 & 14.7 & & & \\
\hline
\end{tabular}




\begin{tabular}{lccccc}
$\ldots / \ldots$ & & & & \\
\hline Ańo legislativo & \# votaciones & $\mathbf{\%}$ & Periodo legislativo & \# votaciones & \% \\
\hline $\mathbf{2 0 1 4}$ & 725 & 11.2 & & & \\
\hline $\mathbf{2 0 1 5}$ & 797 & 12.3 & & & \\
\cline { 1 - 3 } $\mathbf{2 0 1 6}$ & 889 & 13.7 & $2014-2017$ & & 50.7 \\
\cline { 1 - 3 } & 868 & 13.4 & & & \\
\hline Total & $\mathbf{6 4 6 8}$ & $\mathbf{1 0 0}$ & Total & $\mathbf{6 4 6 8}$ & $\mathbf{1 0 0}$ \\
\hline
\end{tabular}

Fuente: elaboración propia con datos extraídos de: www.camara.cl

La variable independiente para la primera hipótesis es el ciclo electoral. Para ello, incluimos indicadores dicotómicos para votaciones realizadas en el primer ańo de gobierno y en el último año de cada cuatrienio. Para el resto de las hipótesis, las variables independientes se relacionan con la temática del proyecto de ley. Estudios previos han categorizado las iniciativas legislativas en diferentes áreas temáticas (Byers et al., 2019). Siguiendo ese principio, para determinar la temática del proyecto usamos como proxy la comisión permanente a la que este fue referido. Las comisiones distribuyen las tareas del poder legislativo, permiten especialización de los miembros y constituyen la forma en que los partidos ejercen su poder de cartelización (Shepsle y Weingast, 1994; Martin, 2014; Krehbiel, 1991; Cox y McCubbins, 1993, 2007). Al ser la instancia que facilita al legislativo cumplir con su tarea de legislar, las comisiones se dividen el trabajo de revisar proyectos de ley a partir de las temáticas de dichos proyectos.

Por cierto, la misma comisión puede tramitar proyectos con orientación ideológica opuesta. Por eso, agrupar todos los proyectos de una comisión presenta el riesgo de diluir las diferencias ideológicas de distintos proyectos revisados por una misma coalición, un problema común a los estudios que utilizan el concepto de issue ownership. La variable independiente para la segunda hipótesis es si el proyecto votado versaba sobre presupuesto y/o si fue referido a la Comisión de Hacienda. Los indicadores de la variable independiente para la tercera hipótesis son si el proyecto fue referido a la Comisión de Defensa o a la de Seguridad Ciudadana y si el proyecto fue referido a alguna comisión encargada de asuntos de protección social. Para ello, identificamos a las Comisiones de Educación, Trabajo, Salud, Familia, Vivienda y Desarrollo Social. Finalmente, la variable independiente para la cuarta hipótesis es si el proyecto fue referido a las comisiones de Derechos Humanos o Constitución.

Siguiendo estudios previos sobre comportamiento legislativo en Chile (Toro 2007; Martínez y Navia, 2019), incluimos como variables de control el tipo de iniciativa (mensaje presidencial o moción parlamentaria), cámara de origen (Cámara de Diputados, Senado), trámite constitucional (primer trámite, posteriores) y el quorum (simple, especiales). Además, incorporamos un dummy para el periodo 2010-2013, para distinguir entre Gobiernos. La tabla 3 muestra los estadísticos descriptivos para todas las variables. 
TABLA 3.

EsTADÍSTICOS DESCRIPTIVOS DE LAS VARIABLES DEPENDIENTES E INDEPENDIENTES

\begin{tabular}{llcccc}
\hline Nombre & $\mathbf{N}$ & Mínimo & Máximo & Media & Desv. est. \\
\hline Unity NM & 6468 & 0 & 1 & 0,68 & 0,24 \\
\hline Rice NM & 6468 & 0 & 1 & 0,84 & 0,25 \\
\hline Unity Alianza & 6468 & 0 & 0,98 & 0,69 & 0,21 \\
\hline Rice Alianza & 6468 & 0 & 1 & 0,90 & 0,22 \\
\hline Unity Oficialismo & 6468 & 0 & 1 & 0,74 & 0,20 \\
\hline Rice Oficialismo & 6468 & 0 & 1 & 0,91 & 0,20 \\
\hline Unity Oposición & 6468 & 0 & 0,98 & 0,63 & 0,23 \\
\hline Rice Oposición & 6468 & 0 & 1 & 0,83 & 0,26 \\
\hline H1 Primer ańo de gob. & 6468 & 0 & 1 & 0,21 & 0,41 \\
\hline H1 Último ańo de gob. & 6468 & 0 & 1 & 0,28 & 0,45 \\
\hline H2 Presupuesto & 6468 & 0 & 1 & 0,19 & 0,40 \\
\hline H2 Hacienda & 6468 & 0 & 1 & 0,08 & 0,27 \\
\hline H3 Defensa & 6468 & 0 & 1 & 0,02 & 0,12 \\
\hline H3 Seguridad & 6468 & 0 & 1 & 0,01 & 0,10 \\
\hline H3 Educación & 6468 & 0 & 1 & 0,14 & 0,34 \\
\hline H3 Familia & 6468 & 0 & 1 & 0,02 & 0,13 \\
\hline H3 Salud & 6468 & 0 & 1 & 0,04 & 0,20 \\
\hline H3 Desarrollo Social & 6468 & 0 & 1 & 0,00 & 0,06 \\
\hline H3 Trabajo & 6468 & 0 & 1 & 0,04 & 0,20 \\
\hline H3 Vivienda & 6468 & 0 & 1 & 0,01 & 0,12 \\
\hline H4 Derechos Humanos & 6468 & 0 & 1 & 0,01 & 0,11 \\
\hline H4 Constitución & 6468 & 0 & 1 & 0,10 & 0,30 \\
\hline Primer trámite & 6468 & 0 & 1 & 0,67 & 0,47 \\
\hline Segundo trámite & 6468 & 0 & 1 & 0,13 & 0,34 \\
\hline Tramites posteriores & 6468 & 0 & 1 & 0,21 & 0,40 \\
\hline Quórum simple & 6468 & 0 & 1 & 0,79 & 0,41 \\
\hline Senado & 6468 & 0 & 1 & 0,14 & 0,35 \\
\hline Mensaje & 6468 & 0 & 1 & 0,19 & 0,40 \\
\hline Periodo 2010-2013 & 6468 & 0 & 1 & 0,51 & 0,5 \\
\hline
\end{tabular}

Fuente: elaboración propia con datos extraídos de www.camara.cl

\section{LA COHESIÓN EN VOTACIONES EN SALA ENTRE 2010 Y 2017}

La figura 1 presenta la cohesión de las coaliciones con los indicadores Unity y Rice. La coalición oficialista es más cohesionada que la de oposición, resultado que se condice 
con lo reportado anteriormente (Toro 2007; Campos-Parra y Navia, 2017; Martínez y Navia, 2019). Para el periodo 2010-2013, la oficialista Alianza fue más cohesionada que la Concertación/NM, mientras que en 2013-2017, la oficialista Concertación/NM tuvo más cohesión. En el Gobierno de Piñera, la cohesión en la Alianza disminuyó en los últimos dos años con el índice Unity, aunque se mantuvo superior que en la Concertación/NM. Con el índice Rice, la Alianza fue más cohesionada como oficialista que la Concertación/NM. En el segundo cuatrienio de Bachelet, la NM fue más cohesionada en el primer año (honeymoon) que en el resto del periodo en ambos indicadores. Si bien la Alianza estuvo menos unida que la NM, su cohesión fue más uniforme durante todo ese periodo. Presumiblemente, el estar compuesta por más partidos que la Alianza incidió en que la Concertación/NM registrara menores índices de cohesión. Con el indicador Unity, el momento de mayor cohesión oficialista fue en 2014, el primer año del segundo Gobierno de Bachelet, mientras que el momento de menor cohesión oficialista fue en el último año de Bachelet.

\section{Figura 1.}

Evolución en cohesión legislativa, Cámara de Diputados de Chile, 20io-20i 8

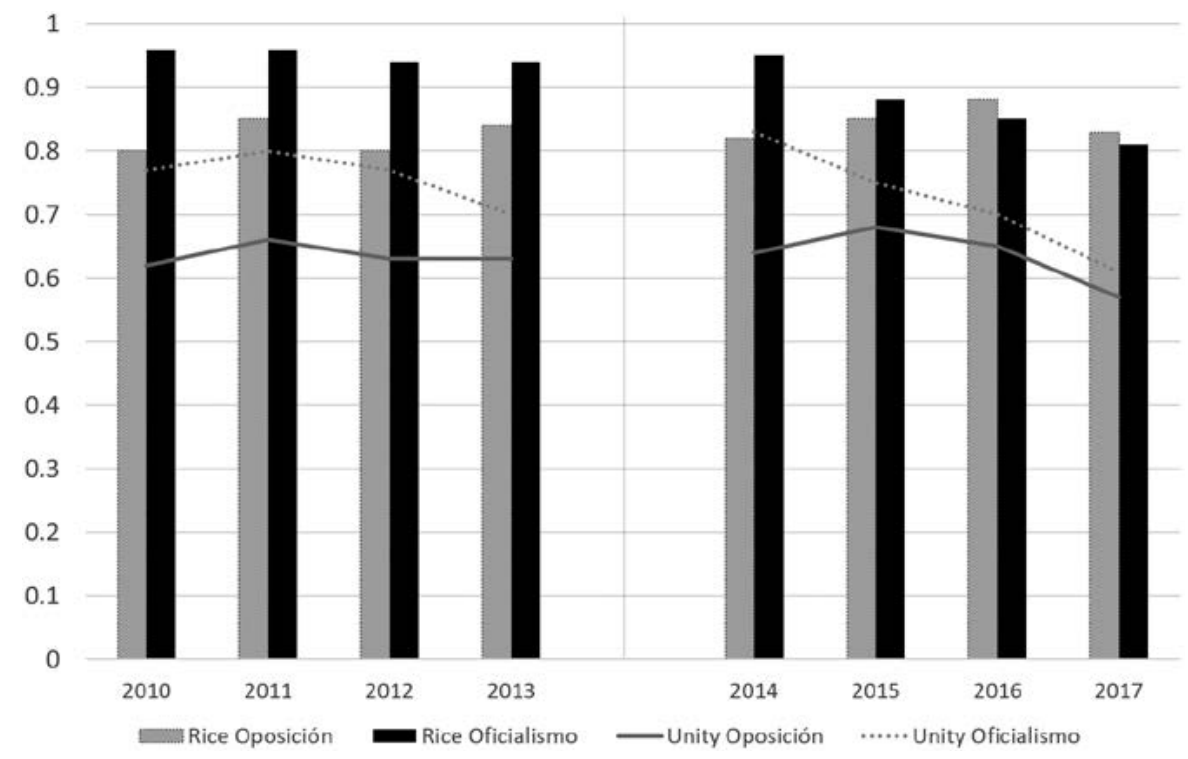

Fuente: elaboración propia con datos extraídos de: www.camara.cl

Ya que la temática de los proyectos de ley es medida por medio de la comisión que tramita cada proyecto, presentamos también información sobre cómo se organiza la legislatura chilena en su trabajo de comisiones. La Cámara tiene veintinueve comisiones permanentes, con trece miembros cada una. A cada partido corresponde una cantidad de cupos en las comisiones proporcional a su número de diputados. Aquí usamos la comisión a la que es referido cada proyecto como proxy de la temática del 
proyecto. La tabla 4 muestra las votaciones según las comisiones a las que fueron referidos los proyectos. El 19,4\% de las votaciones fueron sobre la ley de presupuesto. Las votaciones sobre proyectos tramitados por la Comisión de Educación representan un $13,6 \%$ del total, pero fueron $20,3 \%$ de las realizadas en el segundo Gobierno de Bachelet (marzo de 2014-marzo de 2018), después de que buena parte de su campaña presidencial en 2013 se centró en la educación. Aunque Piñera también debió lidiar con protestas estudiantiles en 2011, el peso relativo de las votaciones de proyectos relacionados con educación en su periodo fue menor. Un 10,2\% de las votaciones se relacionan con proyectos referidos a la Comisión de Constitución, mientras que un $8 \%$ fueron sobre proyectos informados en primera instancia por la Comisión de Hacienda. Por cierto, muchos otros proyectos pasan también por la Comisión de Hacienda, pero aquí consideramos solo la primera comisión a la que fue referido cada proyecto. Los temas de seguridad social también capturan un porcentaje alto de votaciones. Agregados, las votaciones sobre educación (882), trabajo (281), salud (278), vivienda (88), desarrollo social (27) y familia (119) representan el $25,8 \%$ del total en el periodo. Los temas de defensa (102) y seguridad ciudadana (59) representaron solo el $2,5 \%$ de las votaciones.

TABLA 4

Votaciones SEgún la COMisión Revisora del proyecto, Cámara de Diputados de Chile, AÑOS LEGISLATIVOS 2OIO-2OI 7

\begin{tabular}{lcccccc}
\hline Comisión (hipótesis \#) & $\begin{array}{c}\mathbf{2 0 1 0 - 2 0 1 3} \\
(\mathbf{N})\end{array}$ & $\begin{array}{c}\mathbf{2 0 1 4 - 2 0 1 7} \\
(\mathbf{N})\end{array}$ & Total & $\begin{array}{c}\mathbf{2 0 1 0 - 2 0 1 3} \\
\mathbf{( \% )}\end{array}$ & $\begin{array}{c}\mathbf{2 0 1 4 - 2 0 1 7} \\
\mathbf{( \% )}\end{array}$ & Total \\
\hline Presupuesto (H2) & 689 & 567 & 1256 & 21.6 & 17.3 & 19.4 \\
\hline Hacienda (H2) & 253 & 264 & 517 & 7.9 & 8.1 & 8.0 \\
\hline Defensa (H3) & 71 & 31 & 102 & 2.2 & 0.9 & 1.6 \\
\hline Seguridad Ciudadana (H3) & 30 & 29 & 59 & 0.9 & 0.9 & 0.9 \\
\hline Educación (H3) & 216 & 666 & 882 & 6.8 & 20.3 & $\mathbf{1 3 . 6}$ \\
\hline Trabajo (H3) & 98 & 183 & 281 & 3.1 & 5.6 & 4.3 \\
\hline Salud (H3) & 181 & 97 & 278 & 5.7 & 3.0 & 4.3 \\
\hline Familia (H3) & 46 & 73 & 119 & 1.4 & 2.2 & 1.8 \\
\hline Vivienda (H3) & 42 & 46 & 88 & 1.3 & 1.4 & 1.4 \\
\hline Desarrollo Social (H3) & 14 & 13 & 27 & 0.4 & 0.4 & 0.4 \\
\hline Constitución (H4) & 299 & 365 & 664 & 9.4 & 11.1 & 10.3 \\
\hline Derechos Humanos (H4) & $14(*)$ & 68 & 82 & 0.4 & 2.1 & 1.3 \\
\hline Gobierno Interior & 325 & 190 & 515 & 10.2 & 5.8 & 8.0 \\
\hline Economía y Desarrollo & 179 & 93 & 272 & 5.6 & 2.8 & $\mathbf{4 . 2}$ \\
\hline Obras Públicas & 143 & 89 & 232 & 4.5 & 2.7 & 3.6 \\
\hline Pesca & 124 & 31 & 155 & 3.9 & 0.9 & 2.4 \\
\hline & & & & & & $\ldots . .$. \\
\hline
\end{tabular}




\begin{tabular}{lcccccc}
../... & & & & \\
Comisión (hipótesis \#) & $\begin{array}{c}\mathbf{2 0 1 0 - 2 0 1 3} \\
(\mathbf{N})\end{array}$ & $\begin{array}{c}\mathbf{2 0 1 4 - 2 0 1 7} \\
\mathbf{( N )}\end{array}$ & Total & $\begin{array}{c}\mathbf{2 0 1 0 - 2 0 1 3} \\
\mathbf{( \% )}\end{array}$ & $\begin{array}{c}\mathbf{2 0 1 4 - 2 0 1 7} \\
\mathbf{( \% )}\end{array}$ & Total \\
\hline Ciencias y Tecnología & 112 & 15 & 127 & 3.5 & 0.5 & $\mathbf{2 . 0}$ \\
\hline Relaciones Exteriores & 74 & 110 & 184 & 2.3 & 3.4 & 2.8 \\
\hline Minería y Energía & 68 & 81 & 149 & 2.1 & 2.5 & 2.3 \\
\hline Agricultura & 65 & 41 & 106 & 2.0 & 1.3 & 1.6 \\
\hline Medio Ambiente & 58 & 119 & 177 & 1.8 & 3.6 & 2.7 \\
\hline Cultura y las Artes & 27 & 58 & 85 & 0.8 & 1.8 & 1.3 \\
\hline Deportes & 22 & 13 & 35 & 0.7 & 0.4 & 0.5 \\
\hline Desafíos del futuro & - & 20 & 20 & - & 0.6 & 0.3 \\
\hline Régimen Interno & 13 & 4 & 17 & 0.4 & 0.1 & 0.3 \\
\hline Adulto Mayor & 11 & & 12 & 0.3 & 0.0 & 0.2 \\
\hline Turismo & 5 & 3 & 8 & 0.2 & 0.1 & 0.1 \\
\hline Especial de Bomberos & 5 & 3 & 8 & 0.2 & 0.1 & 0.1 \\
\hline Proyectos sin comisión & 4 & & 4 & 0.1 & - & 0.1 \\
\hline R. Hídricos & - & 3 & 3 & - & 0.1 & 0.0 \\
\hline Zonas Extremas & 1 & - & 1 & 0 & - & 0.0 \\
\hline Transparencia & & 3 & 3 & & 0.1 & 0.0 \\
\hline Total & $\mathbf{3 1 8 9}$ & $\mathbf{3 2 7 9}$ & $\mathbf{6 4 6 8}$ & $\mathbf{1 0 0}$ & $\mathbf{1 0 0}$ & 100 \\
\hline
\end{tabular}

Las 14 votaciones sobre tema de Derechos Humanos en el gobierno de Piñera fueron sobre proyectos para otorgar la nacionalidad por gracia a diversas personas.

Fuente: elaboración propia con datos extraídos de: www.camara.cl

La figura 2 muestra la cohesión en las coaliciones de acuerdo con la comisión que revisó cada proyecto. En presupuesto, la NM fue más cohesionada como oposición y la Alianza como oficialismo. Sobre votaciones que implican gasto - Comisión de Hacienda- la cohesión en ambas coaliciones es mayor cuando son oficialismo. En proyectos que pasaron por las comisiones de Defensa y Seguridad, la Alianza fue más cohesionada que la NM, tanto en condición de oficialismo como de oposición. En temáticas sociales, siendo oficialismo y oposición, la NM presenta niveles marginalmente más altos de cohesión que la Alianza. Con relación a salud, los niveles más altos de cohesión se presentaron cuando ambas coaliciones fueron oficialistas. En familia, la Alianza tiende a ser más cohesionada que la NM. En educación, ambas coaliciones fueron más cohesionadas cuando fueron oficialismo. Sobre temáticas sociales, las dos coaliciones presentan niveles más bajos de cohesión respecto a otras temáticas. Sin embargo, ambas presentan el mismo nivel de cohesión siendo oficialismo mientras que, siendo oposición, la Alianza presenta niveles más bajos. Por último, en proyectos que pasaron por la Comisión de Trabajo, la NM tiene mayor cohesión que la Alianza, independiente de su condición de oficialismo u oposición. En cuanto a derechos 
humanos, la Alianza fue más cohesionada al ser oficialismo, mientras que la NM al ser oposición. Ambas coaliciones fueron más cohesionadas en proyectos que pasaron por la Comisión de Constitución cuando fueron oficialismo.

\section{Figura 2.}

COHESIÓN EN LAS VOTACIONES EN SALA DE LAS COALICIONES EN PROYECTOS DE DISTINTAS TEMÁticas, Indicador Unity, Cámara de Diputados de Chile, años legislativos 20Io-20I7
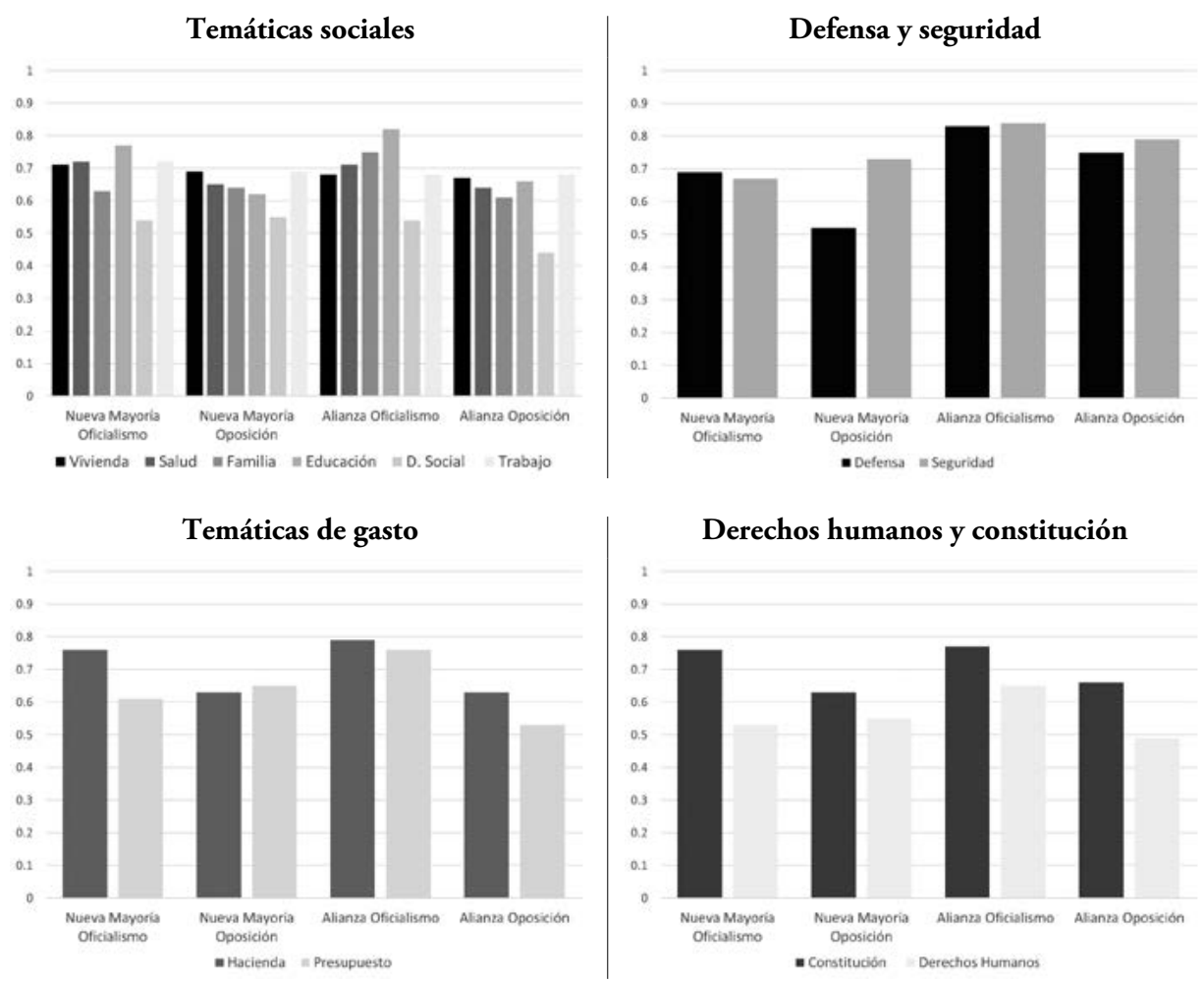

Fuente: elaboración propia con datos extraídos de: www.camara.cl

\section{ANÁLISIS INFERENCIAL}

Para evaluar el efecto de la temática del proyecto de ley sobre la cohesión de las votaciones en sala de los legisladores de las distintas coaliciones, estimamos ocho modelos de mínimos cuadrados ordinarios (MCO). La variable dependiente, cohesión, es medida con los indicadores Unity y Rice y se estima para las coaliciones NM/ Concertación y Alianza, y también para las coaliciones respecto a su condición de oficialismo y oposición en los dos periodos legislativos analizados.

Los modelos de la tabla 5 muestran que, de forma consistente con las expectativas de la hipótesis 1, el oficialismo fue más cohesionado que la oposición en el primer año 
de gobierno y que, en general, hubo menor cohesión en el último año de gobierno en ambas coaliciones. Por su parte, hubo mayor cohesión en la coalición NM/Concertación que en la Alianza. Esto apunta a que la cohesión en la votación en sala de las coaliciones no responde solo a incentivos institucionales o a la condición de oficialismo. De hecho, la Alianza fue menos cohesionada que la NM/Concertación tanto en el primer año de cada periodo legislativo como en el último. Ahora bien, cuando los evaluamos respecto a quién estaba en el poder, el oficialismo fue más cohesionado que la oposición en el primer año, mientras que en el último año ambas coaliciones fueron menos cohesionadas que en el primer año. Pero en el último año, el oficialismo fue menos cohesionado que la oposición.

En contra de lo que postula la hipótesis 2, los modelos no muestran mayor cohesión en el oficialismo que en la oposición en proyectos revisados por la Comisión de Hacienda y la Comisión Especial de Presupuesto. Al contrario, el oficialismo se cohesiona menos en votaciones sobre presupuesto, aunque solo cuando se usa el indicador Unity. Aparentemente, entonces, los legisladores de ambas coaliciones que no quieren votar de forma distinta a cómo lo hará la mayoría de sus pares, prefieren ausentarse de la votación. Eso explica por qué, por ejemplo, hay un efecto positivo de la variable Comisión Especial de Presupuesto en la cohesión de los legisladores de la coalición de oposición cuando se utiliza el indicador Rice. Pero, en general, el efecto de que un proyecto haya sido revisado por la Comisión de Hacienda no es estadísticamente significativo para explicar la cohesión de legisladores del oficialismo y de la oposición —ni de la NM/Concertación o la Alianza-.

Hay una explicación que pudiera dar cuenta de por qué la coalición oficialista se cohesiona menos en temas de presupuesto. Después de todo, el Ejecutivo tiene mayores herramientas para imponer su voluntad en ese tema. Si el Congreso no logra aprobar una ley de presupuesto, automáticamente se promulga la propuesta original de presupuesto presentada por el Ejecutivo. Esa certeza que tiene el Ejecutivo de que se impondrá su voluntad si no hay disponibilidad de negociar pudiera inducir a los legisladores oficialistas —y también a los de oposición— a mostrar sus discrepancias con las posturas dominantes en sus coaliciones, ya sea votando en contra de la posición mayoritaria o simplemente ausentándose de las votaciones sobre presupuesto o sobre proyectos referidos a la Comisión de Hacienda.

Los modelos también son parcialmente consistentes con la tercera hipótesis, que plantea que los legisladores de la coalición de derecha se cohesionan más en votaciones sobre proyectos de ley de defensa y seguridad ciudadana, mientras que los de la coalición de izquierda se cohesionan más en votaciones sobre proyectos de ley en protección social — desarrollo social, vivienda, trabajo, educación, familia y salud—. Cuando se votan proyectos de temáticas de seguridad y defensa, hay mayor cohesión en la Alianza y hay un efecto negativo de la votación en temas de defensa en la cohesión de los legisladores de la Concertación/NM, pero el efecto de proyectos sobre seguridad no tiene significancia estadística. Nótese que la oposición tiende a cohesionarse más en temas de defensa y menos en temas de seguridad, por lo que la mayor cohesión de la derecha en estos temas no tiene que ver con si está en el oficialismo o en la oposición, 
sino con la temática de los proyectos. A su vez, los legisladores de la coalición de centroizquierda no muestran mayor cohesión sistemáticamente en temas asociados con la protección social. Votar proyectos de ley de desarrollo social parece ser una cuestión que induce a conflictos en ambas coaliciones, debido a que estas tienden a ser significativamente menos cohesionadas en ese tipo de votaciones. Las temáticas vivienda y trabajo no impactan sobre la cohesión de las coaliciones. La Concertación/ NM tiende a ser menos cohesionada al votar proyectos de ley sobre familia, pero no la Alianza. Educación tiene un efecto significativo y positivo solo para la cohesión de la Alianza, con los indicadores Unity y Rice. Finalmente, el votar proyectos de ley sobre salud no impacta en la cohesión de las coaliciones.

La cuarta hipótesis sostiene que la coalición de izquierda presenta mayor cohesión que la de derecha en temas de derechos humanos y reforma constitucional. Los datos nos llevan a rechazar esa hipótesis, debido a que este tipo de votaciones produce efecto negativo significativo en la cohesión en la votación de ambas coaliciones. Esto tal vez se explica por el tenor y la cantidad de los proyectos de ley votados en sala. En el periodo marzo de 2010 a marzo de 2014, cuando la centroderechista Alianza fue oficialismo, hubo poco énfasis en votaciones sobre derechos humanos. Estas votaciones se limitaron a proyectos que otorgaban nacionalidad por gracia a distintas personas. Por otro lado, las votaciones de proyectos revisados por la Comisión de Constitución tienen un efecto negativo en la cohesión de la NM bajo el indicador Rice, mientras que para la Alianza no hay efectos significativos. Esto se pudiera deber a que, particularmente en ese periodo, ya se comenzaba a producir un quiebre entre los partidos izquierdistas y centristas de esa coalición. Si bien compartían prioridades de protección social, los partidos de centro e izquierda discrepaban sobre la propuesta de una nueva Constitución impulsada por el Gobierno de Bachelet. Mientras la izquierda empujaba reformas más radicales, el PDC impulsaba reformas más graduales.

El efecto de las variables de control en general es el esperado, aunque algunas de ellas no presentan magnitudes estadísticamente significativas. Cuando se considera la temática del proyecto de ley, esto apunta a que el efecto de las variables de diseńo institucional y la importancia que tiene el proyecto respecto al momento en el proceso legislativo en que se está votando es menor. Por tanto, los estudios sobre cohesión en las votaciones en sala de los legisladores de las distintas coaliciones debiesen incluir la temática de la ley para así precisar de mejor forma los modelos estadísticos. Los modelos también muestran que hay ciertas variables que parecen ser más relevantes para la adscripción ideológica de las coaliciones que para su condición de oficialismo o de oposición. Por ello, las explicaciones que dan cuenta de por qué las coaliciones se cohesionan más en ciertas votaciones que en otras deben incorporar elementos de diseño institucional, como el momento en el periodo legislativo en que se vota un proyecto, la complejidad del proyecto evidenciado en los requisitos de mayorías especiales o cómo de avanzado está el proyecto en el proceso legislativo. Así también, las explicaciones deben incorporar cuestiones asociadas a la condición de oficialismo y a la adscripción ideológica de cada coalición, que hace que ciertos temas generen más cohesión en las respectivas coaliciones. 


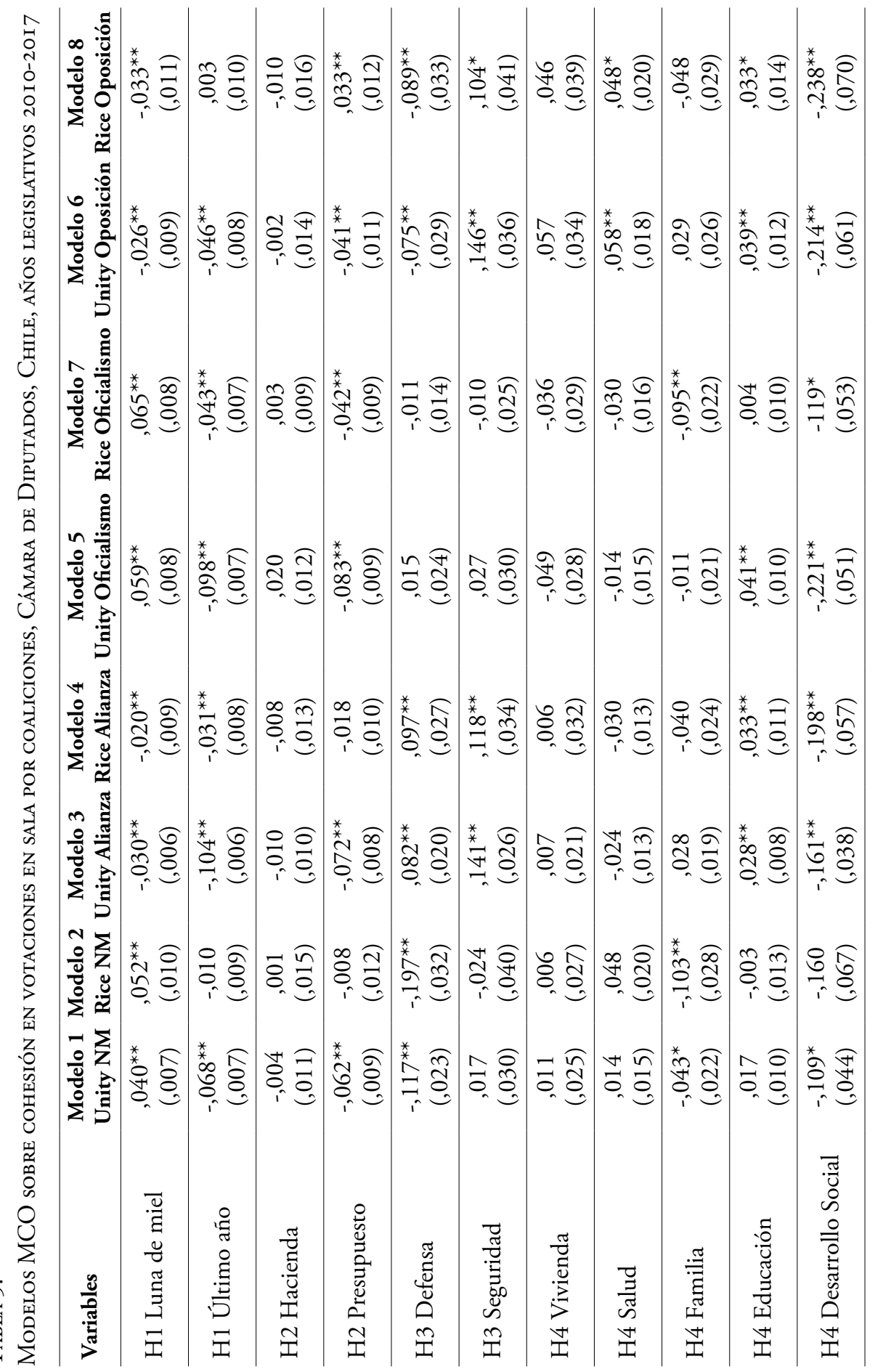




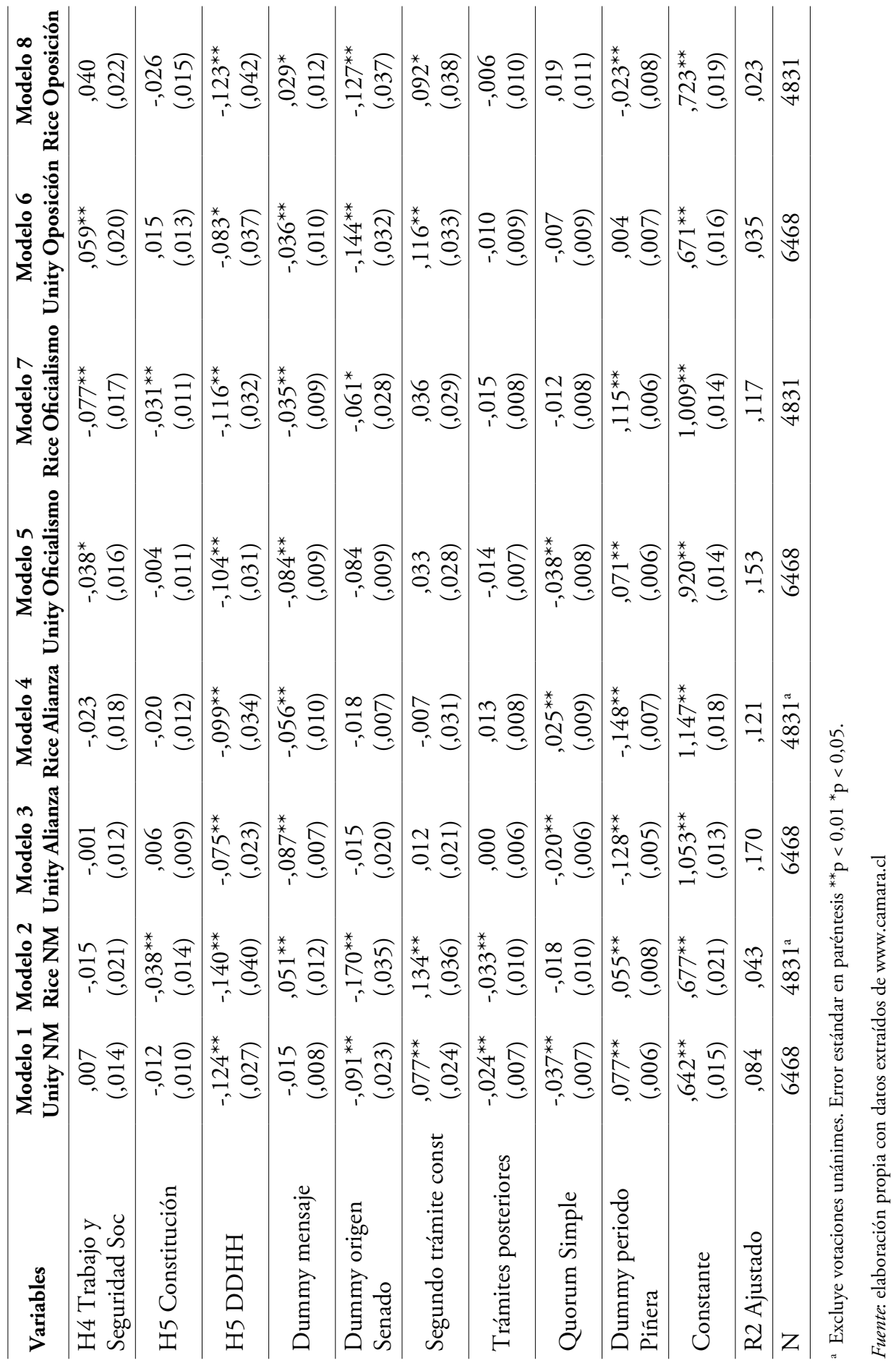




\section{CONCLUSIONES}

A partir de estudios anteriores que apuntan a que la ideología de los legisladores es un determinante de la cohesión partidista (Close 2018; Close y Nuñez 2016), aquí evaluamos el impacto de la temática de los proyectos de ley en la cohesión de la votación de los legisladores de las coaliciones multipartidistas en Chile, un país con un sistema de partidos institucionalizado — a diferencia de otros países de América Latina, que tienen sistemas de partidos más débiles y fragmentados-.

Evaluamos el efecto de la temática del proyecto de ley sobre la cohesión en la votación en sala de los legisladores de las dos principales coaliciones políticas. Si bien ambas coaliciones se cohesionan más en el primer año de gobierno, la cohesión es mayor en el oficialismo en el primer año, pero también cae más en el último año. Los legisladores de la coalición de derecha se cohesionan más en proyectos relacionados con defensa y seguridad ciudadana. Los de la coalición de izquierda no se cohesionan más en temas relacionados con asuntos sociales o derechos humanos. El oficialismo no se cohesiona más en votaciones de presupuesto o en proyectos que pasan por la Comisión de Hacienda. La temática de los proyectos de ley parece explicar mejor la cohesión en la coalición derechista - formada por dos partidos grandes y otros menores- que en la coalición centroizquierdista — formada por seis partidos de tamaño similar- . Si bien la comisión a la que fue referido cada proyecto importa en la cohesión de la votación de los legisladores, parece importar más en los temas en que la derecha tradicionalmente ha ejercido dominancia que en los temas en que la centroizquierda ha ejercido dominancia. Esto apunta a que los temas generan distinta transversalidad en los partidos.

A su vez, al destacar que hay diferencias en la cohesión dependiendo de si se usa el indicador Rice o el Unity, subrayamos que las reglas del juego importan. Ya que Rice solo considera a los legisladores presentes en sala para medir cohesión — mientras que Unity considera que los legisladores ausentes en realidad están votando contra la mayoría del partido - cada indicador mide aspectos diferentes. En países en que las tasas de ausencia de legisladores en votaciones son bajas, ambos indicadores presentarán resultados similares. Pero en países en que los legisladores se ausentan de votaciones por su trabajo en terreno o por otras actividades políticas, el indicador Unity pudiera estar sobrevalorando las diferencias al suponer que los legisladores que no están en sala tienen posiciones divergentes de las que expresan la mayoría de sus correligionarios que si emiten un voto.

Nuestro estudio confirma resultados de estudios anteriores — discutidos en la sección de teoría - que muestran mayor cohesión en el oficialismo, al comienzo de cada periodo y en algunos temas en que los partidos o coaliciones ejercen dominancia. También confirmamos una baja en la cohesión hacia fines del periodo. Además, reportamos que la dominancia sobre ciertos temas - issue ownership - no afecta de igual forma a las delegaciones legislativas de todos los partidos o coaliciones. En Chile, la derecha se cohesiona más en temas en los que ejerce dominancia - como defensa y seguridad ciudadana - , pero los legisladores de centroizquierda no aparecen más cohesionados en temas que normalmente se asocian como dominantes para ese sector. Por 
tanto, parece haber más cohesión en las votaciones de los contingentes legislativos de derecha que de izquierda, independientemente de qué coalición controle el Gobierno nacional. Esto pudiera ser particular en el caso de Chile - y explicado por el número de partidos que forman cada coalición o por asuntos coyunturales que llevaron a la derecha a ser más cohesionada en este periodo-. Pero si suponemos que, al privilegiar la diversidad y las diferencias, la izquierda está más abierta a aceptar distintos puntos de vista, la menor cohesión en la izquierda pudiera ser un asunto generalizable más allá de Chile. Si la derecha a su vez opta por defender tradiciones y por ser valorar más el orden que la diversidad o las diferencias, resultaría lógico esperar que en otros países también haya más cohesión en las votaciones en sala de legisladores de derecha cuando se discuten asuntos relacionados con la diversidad. Aunque cohesión no es antónimo de diversidad o diferencias, si un partido o coalición promueve la diversidad, parecería más difícil lograr que, a la vez, exija cohesión a sus legisladores al votar proyectos que enfrenten al orden con la diversidad. Volviendo a nuestro ejemplo inicial de la votación sobre el aborto en tres causales en Chile, la amplitud de visiones en los partidos de izquierda —algunos de los cuales apoyan el aborto libre, mientras otros apoyan el solo derecho al aborto bajo condiciones restrictivas - pudiera dar cuenta de la falta de cohesión en la forma como votan los legisladores de izquierda en sala.

Con todo, nuestro estudio subraya la importancia de incorporar la temática de los proyectos de ley en las investigaciones sobre comportamiento de los contingentes legislativos de las coaliciones - o en su defecto, de partidos - con presencia en el poder legislativo. Porque la cohesión en la votación de los proyectos de ley varía dependiendo del tema que abarca el proyecto que se vota, las investigaciones sobre el comportamiento del legislativo deberían ir más allá de evaluar la militancia partidista de los legisladores o las reglas del funcionamiento del proceso legislativo e incorporar las temáticas de los asuntos que se discuten. Como hemos mostrado usando el caso de Chile, tanto el oficialismo como la oposición y las coaliciones de izquierda y derecha muestran diferencias en la cohesión de sus legisladores al votar proyectos en sala dependiendo de la temática que aborda el proyecto.

\section{AGRADECIMIENTOS}

Los autores agradecen el apoyo del Fondo Nacional de Desarrollo Científico y Tecnológico (Proyecto \#1200317), los comentarios de Vicente Faúndez Caicedo, Jhon Jaime Godoy, Carolina Pérez Aburto, a los dos revisores anónimos y al equipo editorial de la RECP.

\section{Referencias}

Ahrend, Rudiger, Jens Matthias Arnold, y Charlotte Moeser. 2011. The Sharing of Macroeconomic Risk: Who Loses (and Gains) from Macroeconomic Shocks. OECD 
Economics Departament Working Papers, 877. París: OECD. Disponible en: https://doi.org/10.2139/ssrn.2126666.

Alemán, Eduardo. 2013. «Latin American Legislative Politics: A Survey of Peer-Reviewed Publications in English", Journal of Politics in Latin America, 5 (1): 15-36. Disponible en: https://doi.org/10.1177/1866802X1300500102.

Alemán, Eduardo y Ernesto Calvo. 2008. «Analyzing Legislative Success in Latin America: The Case of Democratic Argentina», en Guillermo O'Donnell, Joseph Tulchin y Augusto Varas (eds.), New Voices in the Study of Democracy in Latin America. Washington D. C.: Woodrow Wilson International Center for Scholars. Alemán, Eduardo y Patricio Navia. 2009. «Institutions and the Legislative Success of "Strong" Presidents: An Analysis of Government Bills in Chile», Journal of Legislative Studies, 15 (4): 401-419. Disponible en: https://doi.org/10.1080/1357233 0903302471.

Alemán, Eduardo y Sebastián M. Saiegh 2007. «Legislative preferences, political parties, and coalition unity in Chile», Comparative Politics, 39 (3): 253-272. Disponible en: https://doi.org/10.2307/20434040.

Allan, James y Lyle Scruggs. 2004. «Political Partisanship and Welfare State Reform in Advanced Industrial Societies», American Journal of Political Science, 48 (3): 496-512. Disponible en: https://doi.org/10.1111/j.0092-5853.2004.00083.x.

Amorim Neto, Octavio. 2000. «Gabinetes presidenciais, ciclos eleitorais e disciplina legislativa no Brasil», Dados, 43 (3): 479-519. Disponible en: https://doi. org/10.1590/S0011-52582000000300003.

Astudillo, Javier y Toni Rodon. 2013. «El comportamiento electoral del votante en la mediana y las "paradojas" de la competición política española", Revista Española de Investigaciones Sociológicas, 144: 3-21. Disponible en: http://dx.doi.org/10.5477/ cis/reis.144.3.

Bélanger, Éric. 2000. "Issue ownership by Canadian political parties 1953-2001», Canadian Journal of Political Science/Revue canadienne de science politique, 36 (3): 539-558. Disponible en: https://doi.org/10.1017/S0008423903778755.

Bélanger, Éric, y Richard Nadeau. 2015. «Issue ownership of the economy: Crosstime effects on vote choice», West European Politics, 38 (4): 909-932. Disponible en: https://doi.org/10.1080/01402382.2015.1039373.

Bobbio, Norberto y Agustín Squella. 1995. «Derecha e izquierda: la igualdad hace la diferencia", Estudios Públicos, 60: 415-429.

Byers, Jason S., Jamie L. Carson y Ryan D. Williamson. 2020. «Policymaking by the Executive: Examining the Fate of Presidential Agenda Items», Congress and the Presidency, 47 (1): 1-31. Disponible en: https://doi.org/10.1080/07343469.2019 .1631905 .

Campos-Parra, Hernán y Patricio Navia. 2017. «Disciplina legislativa en la Cámara de Diputados de Chile, 2010-2014», Politica y Gobierno, 24 (1): 81-123.

Canes-Wrone, Brandice, y Scott De Marchi. 2002. «Presidential approval and legislative success", Journal of Politics, 64 (2): 491-509. Disponible en: https://doi.org/ $10.1111 / 1468-2508.00136$. 
Carey, John. 2002. "Parties, Coalitions, and the Chilean Congress in the 1990s», en Scott Morgerstern y Benito Nacif (eds.), Legislative Politics in Latin America. Nueva York: Cambridge University Press. Disponible en: https://doi.org/10.1017/ CBO9780511615665.009.

Carey, John. 2007. "Competing Principals, Political Institutions, and Party Unity in Legislative Voting», American Journal of Political Science, 51 (1): 92-107. Disponible en: https://doi.org/10.1111/j.1540-5907.2007.00239.x.

Carey, John. 2009. Legislative Voting and Accountability. Nueva York: Cambridge University Press. Disponible en: https://doi.org/10.1017/CBO9780511810077.

Carey, John M. y Matthew Soberg Shugart. 1995. "Incentives to cultivate a personal vote: A rank ordering of electoral formulas», Electoral Studies, 14 (4): 417-439. Disponible en: https://doi.org/10.1016/0261-3794(94)00035-2.

Close, Caroline. 2018. «Parliamentary party loyalty and party family: The missing link?», Party Politics, 24 (2): 209-219. Disponible en: https://doi.org/10.1177/ 1354068816655562.

Close Caroline y Lidia Nuñez. 2016. At the root of parliamentary party cohesion: the role of intraparty heterogeneity and party ideology. Cahiers du CEVIPOL/Brussels Working Papers, 1.

Cox, Gary y Mathew McCubbins. 2005. Setting the agenda: Responsible Party Government in the US House of Representatives. Cambridge, USA: Cambridge University Press. Disponible en: https://doi.org/10.1017/CBO9780511791123.

Cox, Gary y Mathew McCubbins. 2007. Legislative Leviathan: Party Government in the House. Nueva York: Cambridge University Press. Disponible en: https:/doi. org/10.1017/CBO9780511810060.

Cox, Gary y Scott Morgenstern. 2001. «Legislaturas reactivas y presidentes proactivos en América Latina», Desarrollo Económico, 41 (163): 373-393. Disponible en: https://doi.org/10.2307/3456006.

Desposato, Scott W. 2005. "Correcting for small group inflation of roll-call cohesion scores», British Journal of Political Science, 35 (4): 731-744. Disponible en: https:// doi.org/10.1017/S0007123405000372.

Egan, Patrick J. 2013. Partisan Priorities: How Issue Ownership Drives and Distorts American Politics. Nueva York: Cambridge University Press. Disponible en: https://doi.org/10.1017/CBO9781107337138.

Ffrench-Davis, Ricardo. 2002. «Las reformas económicas en América Latina y los desafíos del nuevo decenio", Estudios Internacionales, 35 (138): 7-54. Disponible en: https://doi.org/10.5354/0719-3769.2002.14711.

Figueiredo, Argelina C. y Limongi, Fernando. 2000. «Presidential power, legislative organization, and party behavior in Brazil», Comparative Politics, 32 (2): 151-170. Disponible en: https://doi.org/10.2307/422395.

Freidenberg, Flavia, Fátima García Díez e Iván Llamazares Valduvieco. 2006. «Instituciones políticas y cohesión ideológica. Un análisis multinivel de la heterogeneidad ideológica en los partidos latinoamericanos», en Manuel Alcántara Sáez (ed.). Políticos y política en América Latina. Madrid: Fundación Carolina. 
Fuentes, Claudio. 2015. «Shifting the Status Quo: Constitutional Reforms in Chile», Latin American Politics and Society, 57 (1): 99-122. Disponible en: https://doi. org/10.1111/j.1548-2456.2015.00258.x.

Garretón, Manuel. 2003. Incomplete Democracy. Political Democratization in Chile and Latin America. Chapel Hill, NC: The University of North Carolina Press.

Green, Jane. 2007. «When Voters and Parties Agree: Valence Issues and Party Competition", Political Studies, 55: 629-655. Disponible en: https://doi.org/10.1111/ j.1467-9248.2007.00671.x.

Hazan, Reuven. 2003. «Does Cohesion Equal Discipline? Toward a Conceptual Delineation», The Journal of Legislative Studies, 29 (4): 1-11. Disponible en: https:// doi.org/10.1080/1357233042000306227

Hicks, Alexander y Duane Swank. 1992. "Politics, Institutions, and Welfare Spending in Industrialized Democracies, 1960-82», American Political Science Review, 86 (3): 658-674. Disponible en: https://doi.org/10.2307/1964129.

Hinich, Melvin y Michael Munger. 1996. Ideology and the Theory of Political Choice. Ann Arbor, MI: University of Michigan Press.

Hix, Simon. 2001. «Legislative behaviour and party competition in the European Parliament: An application of nominate to the EU», Journal of Common Market Studies, 39 (4): 663-688. Disponible en: https://doi.org/10.1111/1468-5965.00326.

Janda, Kenneth. 1980. Political Parties: A Cross-national Survey. Nueva York: Free Press. Disponible en: https://doi.org/10.2307/1961087.

Kerlinger, Fred. 1984. Liberalism and Conservatism: The Nature and Structure of Social Attitudes. Hillsdale, NJ: Erlbaum.

Kitschelt, Herbert y Regina Smyth. 2002. «Programmatic party cohesion in emerging postcommunist democracies: Russia in comparative context», Comparative Political Studies, 35 (10): 1228-1256. Disponible en: https://doi.org/10.1177/00104 1402237949.

Korpi, Walter y Joakim Palme. 2003. «New Politics and Class Politics in the Context of Austerity and Globalization: Welfare State Regress in 18 Countries, 1975-95", The American Political Science Review, 97 (3): 425-446. Disponible en: https://doi. org/10.1017/S0003055403000789.

Krehbiel, Keith. 1991. Information and Legislative Organization. Ann Arbor, MI: University of Michigan Press. Disponible en: https://doi.org/10.3998/mpub.8850.

Larraín, Felipe y Rodrigo Vergara (eds.). 2000. La transformación económica de Chile. Santiago: Centro de Estudios Públicos.

Londregan, John. 2000. Legislative Institutions and Ideology in Chile. Nueva York: Cambridge University Press. Disponible en: https:/doi.org/10.1017/CBO9780 511571565.

Mainwaring, Scott y Matthew Shugart. 2002. Presidencialismo y democracia en América Latina. Buenos Aires: Paidós.

Martin, Shane. 2014. "Committees», en Martin Shane, Thomas Saalfeld y Kaare Strom (eds.), The Oxford Handbook of Legislative Studies. Oxford: Oxford University Press. Disponible en: https://doi.org/10.1093/oxfordhb/9780199653010.013.0006. 
Martínez, David y Patricio Navia. 2019. «Determinantes de la cohesión en la votación del legislativo en las coaliciones oficialistas y de oposición en la Cámara de Diputados de Chile, 2006-2014», Revista de Estudios Politicos, 185: 225-258. Disponible en: https://doi.org/10.18042/cepc/rep.185.08.

McClosky, Herbert y John Zaller. 1984. The American Ethos: Public Attitudes toward Capitalism and Democracy. Cambridge: Harvard University Press. Disponible en: https://doi.org/10.4159/harvard.9780674428522.

Meller, Patricio (ed.). 2005. La paradoja aparente. Santiago: Taurus.

Morgenstern, Scott. 2004. Patterns of Legislative Politics: Roll-Call Voting in Latin America and the United States. Nueva York: Cambridge University Press. Disponible en: https://doi.org/10.1017/CBO9780511510328.

Özbudun, Ergun. 1970. Party Cohesion in Western Democracies: A Causal Analysis. Nueva York: Sage.

Petrocik, John. 1996. "Issue ownership in presidential elections, with a 1980 case study", American Journal of Political Science, 27 (3): 825-850. Disponible en: https://doi.org/10.2307/2111797.

Poole, Keith T. y Howard Rosenthal. 2001. "D-nominate after 10 years: A comparative update to congress: A political-economic history of roll-call voting», Legislative Studies Quarterly 26 (1): 5-29. Disponible: https://doi.org/10.2307/440401.

Rice, Stuart. 1925. "The behavior of legislative groups: a method of measurement», Political Science Quarterly, 40 (1): 60-72. Disponible en: https://doi.org/10.2307/ 2142407.

Ruiz Rodríguez, Leticia M. 2006. «Coherencia partidista: la estructuración interna de los partidos políticos en América Latina», Revista Española de Ciencia Política, 14: 87-114.

Schlesinger, Joseph. 1966. Ambition and Politics: Political Careers in the United States. Nueva York: Rand MacNally.

Seeberg, Henrik Bech. 2017. «How stable is political parties' issue ownership? A crosstime, cross-national analysis», Political Studies, 65 (2): 475-492. Disponible en:https://doi.org/10.1177/0032321716650224.

Sehnbruch, Kristen y Peter Siavelis (eds.). 2014. Democratic Chile: The Politics and Policies of a Historic Coalition, 1990-2010. Boulder, CO: Lynne Rienner Publishers.

Sieberer, Ulrich. 2006. "Party unity in parliamentary democracies: A comparative analysis», The Journal of Legislative Studies, 12 (2): 150-178. Disponible en: https:// doi.org/10.1080/13572330600739413.

Shepsle, Kenneth y Barry Weingast. 1994. «Positive Theories of Congressional Institutions», Legislative Studies Quarterly, 19 (2): 149-179. Disponible en: https://doi. org/10.2307/440423.

Stokes, Donald. 1963. Spatial Models of Party Competition», The American Political Science Review, 57 (2): 368-377. Disponible en: https://doi.org/10.2307/1952828.

Stokes, Donald. 1992. "Valence Politics», en Dennis Kavanagh (ed.), Electoral Politics. Oxford: Clarendon Press. 
Stubager, Rune y Rune Slothuus. 2013. "What are the sources of political parties' issue ownership? Testing four explanations at the individual level», Political Behavior, 35 (3): 567-588. Disponible en: https://doi.org/10.1007/s11109-012-9204-2.

Toro, Sergio. 2007. "Conducta legislativa ante las iniciativas del Ejecutivo: unidad de los bloques políticos en Chile», Revista de Ciencia Política, 27 (1): 23-41. Disponible en: http://dx.doi.org/10.4067/S0718-090X2007000200002.

Tsebelis, George. 2002. Veto players: How Political Institutions Work. Princeton, NJ: Princeton University Press. Disponible en: https://doi.org/10.1515/9781400831456.

Van der Brug, Wouter. 2004. «Issue ownership and party choice», Electoral Studies, 23(2):209-233.Disponibleen:https://doi.org/10.1016/S0261-3794(02)00061-6.

Zalaquett, José. 2000. Mesa de Diálogo sobre Derechos Humanos y el Proceso de Transición Politica en Chile. Instituto Nacional de Derechos Humanos. Disponible en: https:// www.indh.cl/wp-content/uploads/2010/10/Mesa_de-Dialogo_CEP2000.pdf.

Presentado para evaluación: 8 de noviembre de 2020.

Aceptado para publicación: 12 de octubre de 2021.

\section{VERÓNICA FIGUEROA-GUTIÉRREZ \\ veronica.figueroa@mail.udp.cl \\ https://orcid.org/0000-0003-2748-1716}

Científica política de la Universidad Diego Portales. Es investigadora asistente del proyecto Fondecyt Regular 1200317 y consultora sobre cuestiones legislativas. Actualmente participa en un proyecto sobre el éxito de las iniciativas legislativas de los miembros de la Cámara de Diputados en Chile entre 1990 y 2018.

\section{PATRICIO NAVIA}

patricio.navia@nyu.edu

https://orcid.org/0000-0001-9398-8393

Doctor en Ciencia Política de la New York University. Es profesor titular de Ciencia Política en la Universidad Diego Portales y profesor titular de Estudios Liberales en la New York University. 